\title{
Research and Application of Fingerprint Recognition Based on Matlab
}

\author{
Ning Liu*
}

Department of Computer Science and Application, Zhengzhou Institute of Aeronautical Industry Management, Henan Zhengzhou 450015, China

\begin{abstract}
With the development of biometric technology, fingerprint recognition technology continues to be widely used in practice due to its uniqueness and reliability. It trends to replace other traditional methods. By using Matlab as a simulation platform and making the best use of its tools, such as powerful graphics and multiple and practical toolbox, etc., the paper makes a series of image processing of the illustration of fingerprint recognition system. Finally, we extract the fingerprint image feature through the experiment.
\end{abstract}

Keywords: Fingerprint image, Matlab, Image Processing.

\section{INTRODUCTION [1-3]}

Under the background of information age, electronic information has a rapid development. And the improvement of the digital and automatic identification is also reflected in personal identification. The network is widely used at present. There are important information security problems, especially, including the information security of personal identity. But our country's various identity management in the area of electronic and digital application is not very perfect. There are a lot of loopholes in recognition. And these loopholes can cause a lot of social problems. However, the method of fingerprint identification can avoid the trouble.

Therefore, fingerprint identification technology is widely used in the field of biological recognition technology and has made a key research at present. The basic schematic diagram of fingerprint identification is shown in Fig. (1).

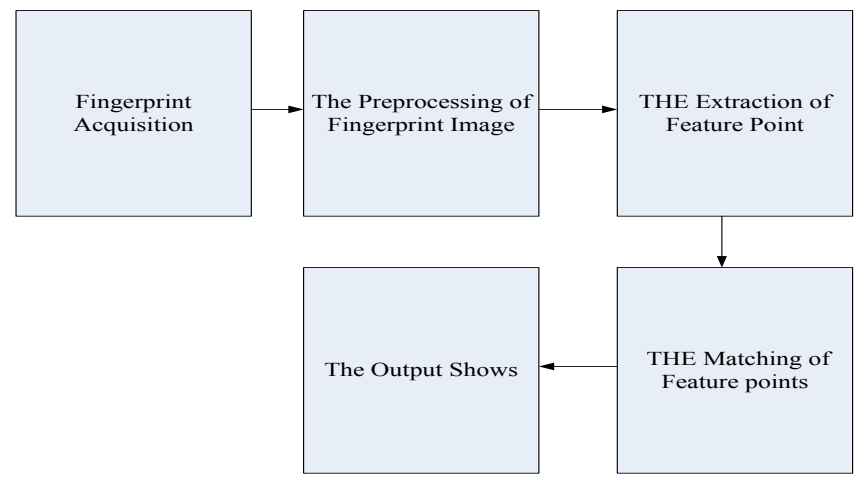

Fig. (1). The schematic diagram of fingerprint identification.

\section{THE PROCESS OF FINGERPRINT IDENTIFICA- TION [4-8]}

The main steps of fingerprint identification technology are shown as the follows.

\footnotetext{
*Address correspondence to this author at the Department of Computer Science and Application, Zhengzhou Institute of Aeronautical Industry Management, Henan Zhengzhou 450015, China; Tel: 15809878998;

E-mail: liuning@163.com
}

(i) Collect the fingerprint image;

(ii) Read the fingerprint image;

(iii) The preprocessing of fingerprint image;

(iv) To extract the feature points;

(v) Save the data and the feature matching.

After the extraction of fingerprint feature points, we will conduct the fingerprint feature point matching. The final results of fingerprint identification is matched through the results. The entire image processing process is shown in Fig. (2).

\subsection{The Matlab Application in Image Processing}

MATLAB has read and displayed function of the image, and the following is a way to read and display grayscale images, that's,

$[X, C m a p]=$ imread, \%(“d: $\backslash$ fingerprint $\backslash$ CASI-A_DBI $\backslash 1 \_1$. bmp); \%Read bmp format file

Cmap; \%Observe color map matrix

Imagesc $(\mathrm{X}) ; \%$ Display grayscale images

Where, $\mathrm{X}$ is a matrix of the gray value of a stored fingerprint image. Gray value image is corresponding to the value of each element within the matrix. To make the image digitized, and the image is processed indirectly through the matrix values. We will observe the result until the image is output by MATLAB.

\subsection{The Fingerprint Image Segmentation}

Fingerprint image segmentation, in the front of the fingerprint image preprocessing, it is one of important and difficult steps in the process of fingerprint image processing. The purpose of image segmentation is to divide the image into several segments has certain significance of sub area or a certain purpose. We can, according to each pixel of image segmentation, still use the image information segmentation within the specified range to complete the image segmenta- 


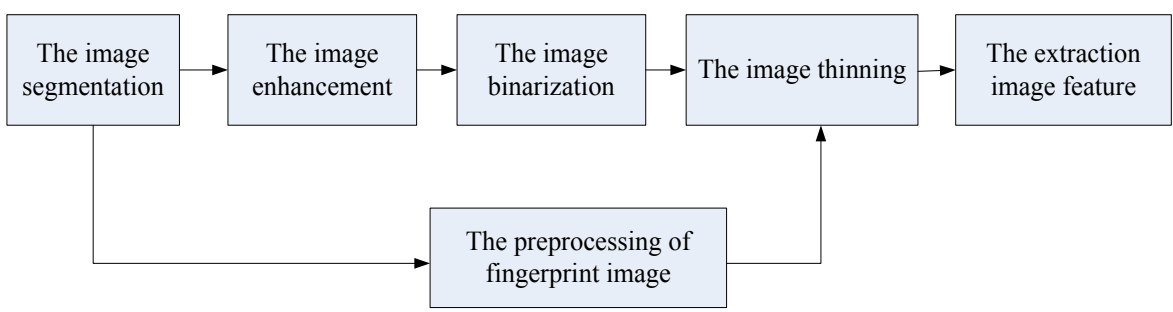

Fig. (2). The flow chart of fingerprint image processing two-dimensional eight shape model.

tion. The fingerprint extraction of the original image is shown in Fig. (3).

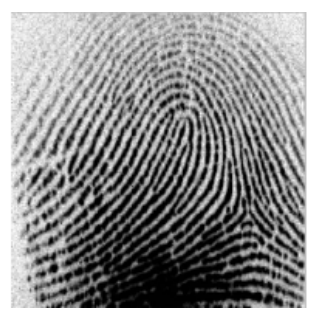

Fig. (3). The original fingerprint image.

The program of reading image is that,

sample=imread('zhiwen.bmp'), \%read image

figure, imshow(sample)

The formula of the fingerprint image processing is given as follows.

$$
G(i, j)=\left\{\begin{array}{l}
M_{0}+\sqrt{\frac{v_{0}}{v_{i}}}\left(I(i, j)-M_{i}\right), I(i, j)>M \\
M_{0}-\sqrt{\frac{v_{0}}{v_{i}}}\left(I(i, j)-M_{i}\right), I(i, j)<M
\end{array}\right.
$$

Where, $M_{0}$ and $M_{\mathrm{i}}$ are the desired mean value, and mean value of fingerprint image respectively. While $v_{0}$ and $v_{i}$ are the desired variance, and variance of fingerprint image respectively.

\subsection{The Fingerprint Image Enhancement}

After the fingerprint image segmentation, we need to make a filter processing in order to eliminate the interference of noise on the image processing. Finally, it will further improve the efficiency of fingerprint image recognition.

The fingerprint image enhancement is usually used to process the image which has a low quality and obscure effect. Therefore, it will let the structure and texture of fingerprint more clear and eliminate some false feature information. Finally, it will retain some key feature information for the fingerprint recognition, which can make the fingerprint identification more accurate and reliable.

In this paper, we combine the median filtering with the high pass filtering technology to process the fingerprint image.
Where, median filtering has some important feature as follows.

(i) One dimensional median filter can maintain the invariance of some certain types of input signals, such as the series of monotone increasing or reducing or the step series. The output signal of median filtering is constant.

(ii) The denoising performance of median filter. When the image has random interference and pulse interference, median filter can reduce the noise interference. This is the superiority on the denoising performance of median filter.

(iii) $M\{f(i, j)\}$ is used to express the median of $f(i, j)$ which is one-dimensional or two-dimensional. Set $C$ as a constant. Then the following equation is true.

$$
\begin{aligned}
& M\{C * f(i, j)=C * M f(i, j)\} \\
& M\{C+f(i, j)=C+M\{f(i, j)\}\} \\
& M\{f(i, j)+g(i, j) \neq M\{f(i, j)\}+M\{f(i, j)\}
\end{aligned}
$$

Using $3 \times 3$ median filtering template to process the fingerprint image in Fig. (3), we can get the Fig. (4) below.

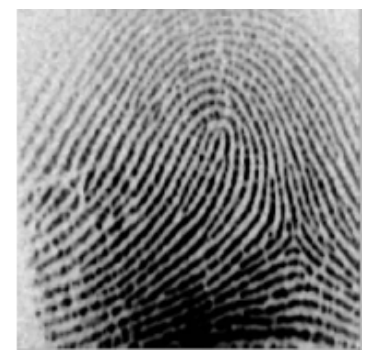

Fig. (4). The fingerprint image after median filtering.

The program of reading image is that, smooth $=$ medfilt 2 (sample, $[3,3])$

figure, imshow(smooth)

High pass filtering is usually carried out after the median filtering. High pass filtering can enhance the high frequency component of image without changing the low frequency components, while the general means will weaken the low frequency components when the high frequency component of image is enhancement. High pass filter can enhance the edge of fingerprint image. It is the basis for the realization of fingerprint image edge detection. The smooth information in 
fingerprint image is substantially suppressed by high pass filter. And it can make the sharpening of image's basic information.

The convolution template in the experiment is $H_{1}$, that's,

$H_{1}=\frac{1}{6}\left[\begin{array}{lll}-1 & -1 & -2 \\ -1 & 17 & -1 \\ -2 & -1 & -1\end{array}\right]$

Take $H_{1}$ template for example, the Fig. (5) and Fig. (6) are shown as follows, respectively.

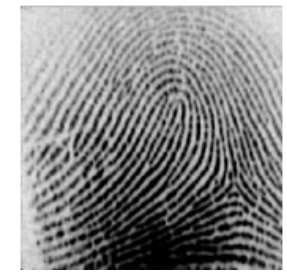

Fig. (5). The fingerprint image before high pass filtering.

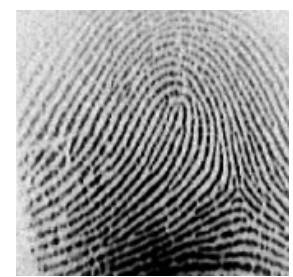

Fig. (6). The fingerprint image after high pass filtering.

\subsection{The Binarization of the Fingerprint Image}

After binarization, it can reduce a lot of useless information for fingerprint identification, and it can be classified according to the enhanced characteristic information at the same time. Therefore, the binarization of the fingerprint image can improve the efficiency of recognition. The theory of image's binarization is as follows. Set a grey level as the threshold in image gray scale. They are recorded as input image $f(i, j)$ and output image $F(i, j)$, respectively. The single threshold segmentation algorithm is as follows.

$F(i, j)=\left\{\begin{array}{l}1, f(i, j)>T \\ 0, f(i, j) \leq T\end{array}\right.$

For the binarization of the fingerprint image, we need to find a suitable threshold through graythresh function. When a gray image is converted into the binary image by the function im $2 b w$, it's mainly to get a suitable threshold by the function graythresh. The threshold which is found by the maximum class variance is more accurate than the factitious setting. In this paper, we use the former to make a gray image change into binary image. The Fig. (7) is the fingerprint image before the binary processing while the Fig. (8) is that after the binary processing.

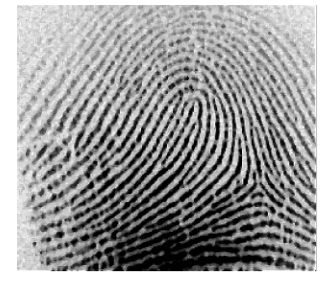

Fig. (7). The fingerprint image before binarization.

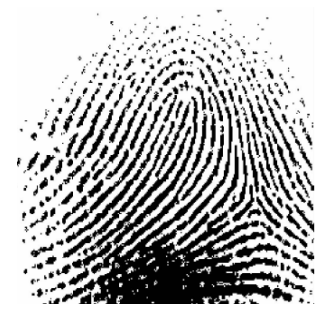

Fig. (8). The fingerprint image after binarization.

It's the binarization program below.

level=graythresh(sharp), \%Calculate the binarization threshold

$\mathrm{bw}=\mathrm{im} 2 \mathrm{bw}$ (sharp,level), \%the binarization image

figure, imshow(bw)

To make a further processing, such as to fill the small holes in the image streaklines, to make the fuzzy region in the binary image clear and remove the pores and cavity fill, we can get the fingerprint image Fig. (9) and Fig. (10) as shown below.

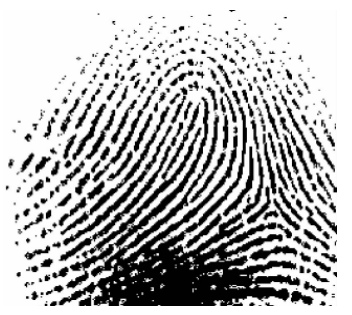

Fig. (9). The fingerprint image before modifying.

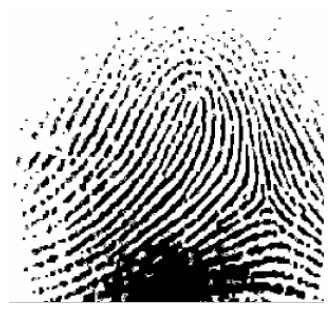

Fig. (10). The fingerprint image after modifying.

The program of removing pores and cavity fill is given as follows.

decorated=decorate(bw);\% Modify the image

figure,imshow(decorated)

\subsection{The Thinning of the Fingerprint Image}

After binarization, the image information doesn't exaggerate the trend of streamline. And the streamline has a larger width. It's not conducive to extract the feature infor- 
mation. Therefore, the image thinning is very important. After thinning, the fingerprint image can reduce some useless information to exaggerate its feature information. The thinning is used to delete the edge pixel of the streamline and make streamline to be single pixel. Finally, it will repair the thinning streamline. Fig. (11) is changed to be Fig. (12) after thinning.

The thinning program is as follows.

thinned=thin 4 (decorated), \%the thinning image

figure, imshow(thinned)

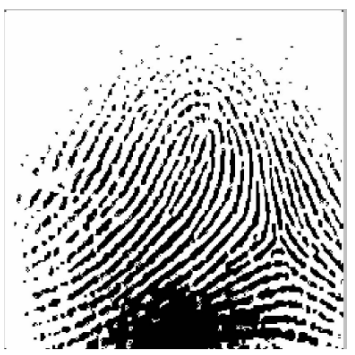

Fig. (11). The fingerprint image before thinning.

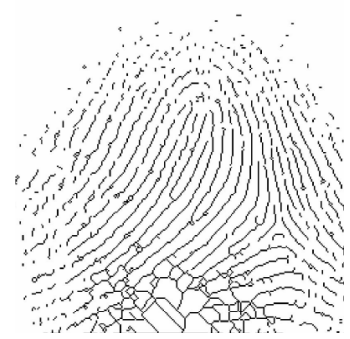

Fig. (12). The fingerprint image after thinning.

\subsection{Acquiring Fingerprint Feature Point Based on MATLAB}

The extraction of fingerprint image feature point is to find the confirmation message with uniqueness. That the extraction of feature point is good or will not affect the final results of the fingerprint matching. We adopt the thinning image after binarization in this paper.

The method of feature point's extraction is that $M n(P)$ is set to be the crossing number and $\operatorname{Sn}(P)$ is pixel 8 neighborhood. They are shown as Fig. (13) and Fig. (14) below.
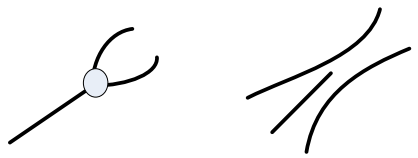

Fig. (13). The Intersection Point and Endpoint.

\begin{tabular}{|c|c|c|}
\hline P 3 & P 2 & P 1 \\
\hline P 4 & P & P 0 \\
\hline P 5 & P 6 & P 7 \\
\hline
\end{tabular}

Fig. (14). The 8 neighborhood of $\mathrm{P}$.

$$
\begin{aligned}
& \operatorname{Mn}(P)=\frac{1}{2} \sum_{i=1}^{8}\left(P_{i-1}-P_{i}\right)\left(P_{9}=P_{1}\right) \\
& \operatorname{Sn}(P)=\sum_{i=1}^{8} P_{i}
\end{aligned}
$$

After thinning completely, fingerprint image has three streaklines points, that's, endpoint, combo point and intersection point. Where, when $\operatorname{Mn}(\mathrm{P})=1, \operatorname{Sn}(\mathrm{P})=3$, they are called the endpoints; when $\operatorname{Mn}(\mathrm{P})=2, \operatorname{Sn}(\mathrm{P})=2,3$, 4, they are called the combo points; when $\operatorname{Mn}(\mathrm{P})=3, \mathrm{Sn}(\mathrm{P})=3$, they are called the intersection points.

Assume that $\mathrm{P}(\mathrm{P} 1, \mathrm{P} 2, \ldots, \mathrm{Pn})$ is used to express a set of the feature points. Where, $\mathrm{Pi}=(\mathrm{Xi}, \mathrm{Yi}, \mathrm{Ti}, \mathrm{Ai})$, and $\mathrm{Xi}, \mathrm{Yi}$ are used to express the coordinates of the feature points, respectively; $\mathrm{Ti}$ is used to express the type of the feature points; $\mathrm{n}$ is the number of feature points. When the feature points are the endpoints, $\mathrm{Ti}=1$, while they are the intersection points, $\mathrm{Ti}=2$.

\subsection{The Experimental Procedure and the Result}

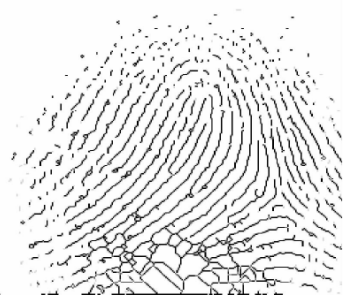

Fig. (16). The extraction of fingerprint image feature.

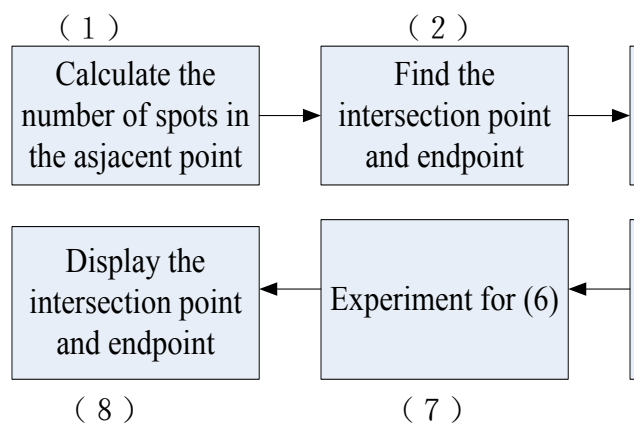

( 3 )

\section{Remove edge effect} Record three black
asjacent point of the
initial bifurcation
point

( 6 )
(4)

Remove breakpoint Remove flash and holes and so on

Fig. (15). The experimental procedure. 
The extraction program of fingerprint image feature is given below Fig. (15) and Fig. (16).

minutiae $=$ feat(thinned), \%search for feature point include endpoint and bifurcation point

figure, imshow(minutiae)

\section{CONCLUSION}

In this paper, the image segmentation, image enhancement, image binarization, image thinning and the extraction of fingerprint image feature point have been analyzed systemically for based on Matlab.

\section{ACKNOWLEDGMENT}

This work is supported by the basic and frontier technology research project of Henan and Technology Department (132300410186) and the basic research project of Henan and Technology Department (2014B520067).
[1] R. C. Hum, "An identification in information systems: management challenges an public policy issues," Info Techno People, vol. 7, no. 04, pp. 6-37, 1994.

[2] Z. Chen, and C.H.Kuo, "A topology-based matching authentication," IEEE, vol. 91, 1991.

[3] N. K. Ratha, S. Y. Chen, and A. K. Jain, "Adaptive flow orientation-based feature extraction in fingerprint images," Pattern Recognition, vol. 28, no. 10, pp. 1657-1672, 1995.

[4] J. Y. Guo, Q. Wu, and Q. R. Sang, "The detail features extraction of fingerprint digital image based on Matlab implementation," Computer Simulation, vol. 24, no. 01, pp. 182-185, 2007.

[5] T. Min, "The research and application of universal fingerprint image processing and analyzing platform," Computer Engineering And Design, vol. 31, no. 04, pp. 791-794, 2010.

[6] W. H. Han, "The preprocessing technology in the automatic fingerprint identification system," Computer Research and Development, vol. 34, no. 12, pp. 913-920, 1997.

[7] H. F. Li, "The research of adaptive binarization algorithm for the fingerprint image based on directed graph," Beijing Normal University (Natural science edition), vol. 45, no. 03, pp. 250-253, 2009.

[8] J. M. Su, "Matlab 7.0 practical guide," Beijing: Electronic Industry Press, 2004.

\section{REFERENCES}

Received: May 26, 2015

Revised: July 14, 2015

Accepted: August 10, 2015

(C) Ning Liu; Licensee Bentham Open.

This is an open access articles licensed under the terms of the Creative Commons Attribution-Non-Commercial 4.0 International Public License (CC BY-NC 4.0) (https://creativecommons.org/licenses/by-nc/4.0/legalcode), which permits unrestricted, non-commercial use, distribution and reproduction in any medium, provided that the work is properly cited. 